\section{ambient \\ SCIENCE \\ Vol. 05(Sp1):60-67 \\ Year 2018}

\title{
Comparative Evaluation on Lipid Profile between Diabetic and Non- diabetic Patients, Signalizing some Common Physiological Complications Intended towards Diabetes: a Case Study from Raipur, C.G., India
}

\section{Vishwaprakash Roy ${ }^{1,2}$, Jayant Biswas ${ }^{2 *}$, Ashish Saraf ${ }^{1}$}

'School of Biological and Chemical Sciences, MATS University, Raipur, Chhattisgarh, India

${ }^{2}$ Central laboratory, National Cave Research and Protection Organization, Raipur, Chhattisgarh, India

Study Area: Raipur, India

Coordinates: $21.25^{\circ} \mathrm{N} ; 81.63^{\circ} \mathrm{E}$

Key words: Diabetes mellitus, Triglycerides, Cholesterol, LDL, VLDL

\section{Abstract}

A Study conducted over a time span of two years on 304 subjects (152, non-diabetic and 152 diabetics) ended with some interesting outcome possibly due to the existing lifestyle of Raipur. During the study, by keeping the male and female in a ratio of 1:1. All the 304 subjects were divided into 4 different age groups. There fasting blood samples were tested for some biochemical parameters viz., plasma glucose and serum lipids. The diabetic patients showed a significant increasing trend in the values of Total Cholesterol, Triglycerides, VLDL and LDL and a significant decrease in the values of HDL as compared to that of nondiabetic subjects. The study showed the prevalence of hyperlipidemic conditions associated with diabetic patients whose intensity and horrific consequences are increasing along with age. The disorders related to lipid parameters occurred more in diabetic females perhaps indicating that the diabetic females of the area are more prone towards the development of cardiovascular diseases in the future. The possible reason behind each unusual findings have been tried correlate with the living style of the area.

utilization of lipids and lipoproteins (Krauss, 2004; Farmer, 2007). Diabetes affects virtually all lipids lipoproteins, resulting dyslipidemia. Patients get typically increased Triglycerides in plasma concentrations causing a low level of 'high-density lipoprotein' (HDL) cholesterol, but slightly raised in 'low-density lipoprotein' (LDL) cholesterol (Ozder, 2014; Schofield et al., 2016). Patients with type 2 diabetes are also prone to a preponderance of atherogenic small dense LDL (Turner et al., 1996; Austin \& Edwards, 1996). The prolong hyperglycemia of Type 2 diabetes usually leads to long-term damage, dysfunction or failure of various organs such as eyes, kidneys, nerves, heart, and blood vessels etc.

Early diagnosis of diabetes could help to predict the risk for the development of a variety of diseases and complications for future in any patients (Khaw et al., 2004). Thus, the physicians generally, prescribe for lipid profile tests to whom they think prone to diabetes. In the present study, an attempt was taken to compare the lipid prof iles of such patients of Raipur, C.G., India who were suffering from some common physiological disorders, referred for serum biochemical tests and diagnosed either as Diabetic or Non- 
diabetic (but might be other disease).

\section{Methodology:}

In the present study, we randomly selected Lipid Profile Test's records of a total 304 subjects belonging to both nondiabetic, and diabetic patients who were prescribed for the same by some authorized physicians of Raipur C.G., India during the period of March 2014 to March 2016. Out of 304 patients (within the age group of 35-75 years), 152 patients were with normal blood glucose level ( 76 males and 76 females) and 152 diabetic patients (76 males and 76 females) with abnormal blood sugar level were considered. Based on age and health conditions, four groups were formed viz., Group-1 (G1) included ages between 35-45 yr., Group 2 (G2) 46-55 yr., Group 3 (G3) 56-65 yr. and Group 4 (G4) 66-75 years. The study was based on the evaluation of the blood sample of such patients who were advised by the physician to diagnose various such blood serum parameters usually referred to diabetes suspected persons.

The standard reference value of fasting blood glucose level used by the physicians was taken as a reference parameter to differentiate the patients as diabetic and nondiabetic.

Sample collection: for the present the study, venous blood samples in fasting state of patients were drawn by the trained laboratory technicians and/or nurses with sterile syringes. Based on biochemical parameter of the blood to be determined, the samples were collected in the non-vacuum tubes of variable specifications as follows:

1. For separation of plasma, tubes containing sodium fluoride were used for determination of values blood glucose from the blood sample.

2. For separation of serum, plain tubes were used to determine the various parameters of lipid profile.

Separation of serum: after collection of blood samples the complete blood was allowed to clot at room temperature by keeping the tubes in a steady and standing position for few minutes. Further, the cells and clot were removed by the process of centrifugation. The supernatant obtained i.e. serum was transferred to a clean test tube for analysis of lipid profile.

Equipment and disposables used: Semi auto blood biochemistry analyzer, Micro-Pipettes, Bench top laboratory centrifuge, Non-vacuum tubes containing sodium fluoride and plain tubes, Internal quality control serum, Antiseptic swabs, Sterile syringes, Kits for determination of blood glucose and lipid prof ile.

Determination of biochemical parameters:

i).Blood glucose: glucose for diabetic and non-diabetic patients was determined by GOD-POD method, End Point ii. Lipid profile: lipid profile tests for diabetic and nondiabetic patients were based on the determination of following parameters: a. Cholesterol: Cholesterol tests for diabetic and non-diabetic patients was done by CHOD-PAP method, End Point.

b. HDL-C: High Density Lipoprotein- Cholesterol test for diabetic and non-diabetic patients was done by End Point method.

c. Triglycerides: Triglycerides test for diabetic and non-diabetic patients was done by GPO-PAP method, End Point

d. LDL-C: Low Density Lipoprotein Cholesterol test for diabetic and non-diabetic patients was directly determined by Friedewald's formula- LDL-C (mg/dl) = TC (mg/dl)- HDL-C (mg/dl)-TG (mg/dl)/5 (Friedewald et al., 1972; Grundy et al., 2005; Nigam, 2014; Dansethakul et al., 2015; Sudha et al., 2015.)

e. VLDL-C: Very Low Density Lipoprotein- Cholesterol was estimated from dividing the values of triglyceride concentration by 5 .

VLDL-C (mg/dl) = TG (mg/dl) / 5 (Friedewald et al., 1972; Grundy et al., 2005; Nigam, 2014; Dansethakul et al., 2015; Sudha et al., 2015.)

Time to time commercially available quality control serum was procured and was analyzed as samples to check the performance of the test and equipment. After the completion of the biochemical analysis of the same, the remaining quality control serum and test samples in the disposable tubes were handed over to the pathology lab for proper treatmentand disposal.

Statistical analysis: to analyze the gender and age effects against each analyzed parameter we used only student's " $\mathrm{t}$ " tests separately.

\section{Results and Discussion:}

Results are summerized in Tables 1 \& Figure-1 to 10

(In each figure, bars having similar superscripts do not differ from each otherat $95 \%$; t-test)

\section{Parameter: Fasting Blood Glucose (mg/dl)}

Gender and various age-dependent comparative Fasting Blood Glucose (mg/dl) values for Non-diabetic Patients: irrespective to the age and gender groups the values of Fasting blood glucose were found to fluctuate in between $(77.8 \pm 0.39$ to $93.6 \pm 0.32 \mathrm{mg} / \mathrm{dl})$. In both the genders, the FBG values were found to reveal an increasing trend with respect to the increasing age-groups ( $\mathrm{G}_{1}$ to 4 ). Further, the FBG value was found to be in its lowest level at the earlier age-groups (G-1) and at its highest in the latter age-groups (G-4). Irrespective to gender differentiation, each age groups statistically differ from its consecutive age-groups. Interestingly, for the two distinct age groups ( $\mathrm{G}-2$ \& 4), the FBG values in the male and female groups were also found to differ statistically from each other.

Gender and various age-dependent comparative Fasting Blood Glucose (mg/dl) values for Diabetic Patients: irrespective to the age and gender groups the values of Fasting blood glucose were found to fluctuate in between (128.2 \pm 1.02 to $178.1 \pm 0.92 \mathrm{mg} / \mathrm{dl})$. The overall results almost reflect the same trend as it revealed in its non-diabetic counterpart. In both the genders, the FBG values were 
found to reveal an increasing trend with respect to the increasing age-groups (G-1 to 4). Statistically, each group differs significantly from its consecutive age-groups. Surprisingly, in the latter age groups (G-3 \& 4), the FBG values in the male and female group were also found to differ statistically from each other.

Comparative Fasting Blood Glucose (mg/dl) values for Non-diabetic versus Diabetic Patients (Male): concluding the above results' pattern, irrespective to the various agegroups, the FBG values were always revealed high in the diabetic patients as compared to their non-diabetic counterparts and were statistically differing at each agegroup. However, in both the groups the values of the parameter were found to increase with respect to the agegroups and which differ statistically from each other.

Comparative Fasting Blood Glucose (mg/dl) values for Non-diabetic Versus Diabetic Patients (Female): in the case of female also the same picture reflected which has been revealed in the case of its male counterpart.

Table-1:. Demographic study of subjects selected for study in Raipur region and their Blood Sugar Level tests' record

\begin{tabular}{llllll}
\hline Para & \multicolumn{4}{l}{ Sex } & \multicolumn{4}{c}{ Age groups $(\mathrm{n}=76)$} \\
meters & & $35-45(\mathrm{G})$ & $46-55\left(\mathrm{G}_{2}\right)$ & $56-65\left(\mathrm{G}_{3}\right)$ & $66-75\left(\mathrm{G}_{4}\right)$ \\
\hline NDG $^{*}$ & $\mathrm{M}$ & $40.15 \pm 0.37$ & $52.28 \pm 0.48$ & $61.57 \pm 0.43$ & $72.35 \pm 0.35$ \\
& $\mathrm{~F}$ & $40.39 \pm 0.62$ & $50.12 \pm 0.38$ & $56.87 \pm 1.79$ & $71.04 \pm 1.02$ \\
$\mathrm{DG}^{*}$ & $\mathrm{M}$ & $40.96 \pm 1.25$ & $50.15 \pm 0.23$ & $62.06 \pm 0.26$ & $77.25 \pm 0.72$ \\
& $\mathrm{~F}$ & $42.22 \pm 0.82$ & $50.15 \pm 1.15$ & $60.09 \pm 0.50$ & $71.10 \pm 0.49$ \\
NDG $^{* *}$ & $\mathrm{M}$ & $77.8 \pm 0.39$ & $84.6 \pm 0.14$ & $88.2 \pm 0.11$ & $91.1 \pm 0.21$ \\
& $\mathrm{~F}$ & $78.9 \pm 0.55$ & $82.5 \pm 0.32$ & $88.0 \pm 0.20$ & $93.6 \pm 0.32$ \\
$\mathrm{DG}^{* *}$ & $\mathrm{M}$ & $128.2 \pm 1.02$ & $137.9 \pm 0.09$ & $149.2 \pm 0.02$ & $161.4 \pm 1.21$ \\
& $\mathrm{~F}$ & $129.8 \pm 1.56$ & $137.2 \pm .71$ & $157.8 \pm 0.03$ & $178.1 \pm 0.92$ \\
\hline
\end{tabular}

*- Age; **-FBG (mg/dl); NDG- Non-diabetic Group; DG- Diabetic Group; M-male; F-female.

The observed values for this parameter were seen to be under control for each age and gender groups in nondiabetic patients. However, little increasing trends were apparent which could be stated as obvious as with increasing age the irregularities in urban lifestyle also increase. Such factors perhaps imbalance the hormonal activities resulting in the increment of Fasting Blood Glucose in Non-diabetic patients too. Universally, the studies support that frequencies of diabetics in urban population is constantly rising (Motala et al., 2003; Gupta et al., 2007; Ko et al., 2006). However, the FBG in females were seen to increase more as compared to the respective male counterparts, it supports various other studies (Hussain et al., 2007; Ghadge et al., 2017; Veghari et al., 2014). However, Ghazanfari et al. (2010) didn't report any notable difference between male and female and with respect to age-groups.

Cholesterol: Reference range: $<200 \mathrm{mg} / \mathrm{dl}$

Results are summerized in Fig-1a,b \& 2a,b

Gender and various age-dependent comparative total cholesterol (mg/dl) for non-diabetic Patients: irrespective to the age and gender groups the values of total cholesterol were found to fluctuate in between $(149 \pm 0.83$ to $231 \pm 1.11$ $\mathrm{mg} / \mathrm{dl})$. However, in both the genders total cholesterol values revealed an increasing trend with respect to the increasing age (G-1 to 4). Statistically irrespective to age, each group differed significantly from its consecutive groups. Further, except for the age-group- 3 in remaining all the age-groups statistically signif icant differences between gender groups were also revealed.

Gender and various age-dependent comparative total cholesterol (mg/dl) for diabetic patients: irrespective to the age and gender groups the values of Total Cholesterol were found to fluctuate in between $(200 \pm 0.95$ to $297 \pm 1.05$ $\mathrm{mg} / \mathrm{dl}$ ). With respect to different-gender groups, the values of Total Cholesterol revealed an increasing trend with respect to the increasing age-groups (G-1 to 4). Statistically, each age, as well as gender group, differed significantly from its consecutive and respective groups.

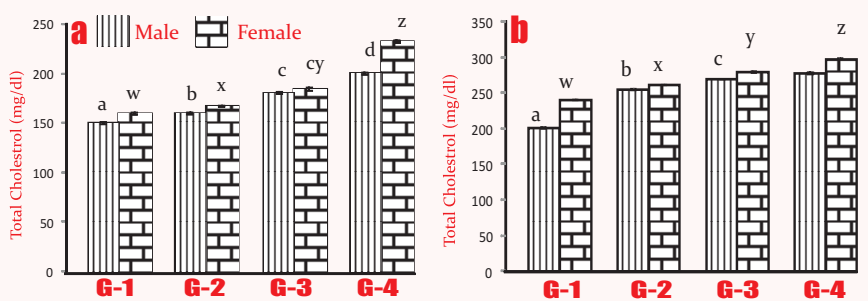

Figure-1: Sex and various age dependant comparative Cholestrol for Non-diabetic (a) and diabetic (b) Patients.

Comparative total cholesterol $(\mathrm{mg} / \mathrm{dl})$ for non-diabetic versus diabetic patients (Male): for both the groups the total cholesterol values were found to raise continuously with respect to the increasing age-groups ( $G-1$ to 4 ) and were also statistically differed from their respective age and gendergroups.

Comparative total cholesterol (mg/dl) for non-diabetic versus diabetic patients (Female): the obtained values for total cholesterol (mg/dl) for female reflect almost the same as its male counterpart for diabetic patients.
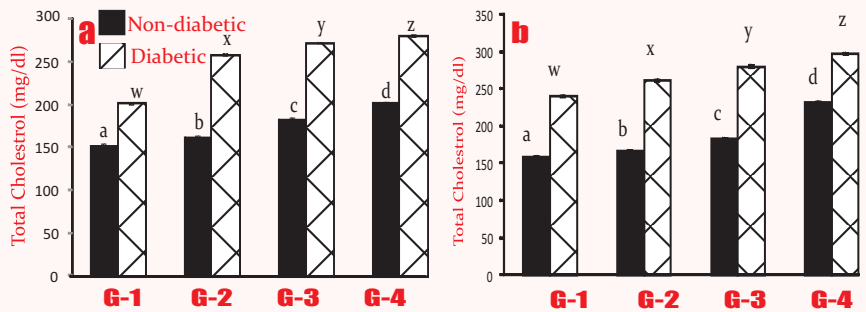

Figure-2: Comparative Total Cholestrol for Non-diabetic Versus Diabetic Patients (Male) (a) and (Female) (b)

Cholesterol level was reported to be high in diabetic patients (Mahendran et al., 2013) and also in non-diabetic obese population (Songa et al., 2013). This parameter was always found to be high in female age-groups than male irrespective to the diabetic or non-diabetic patients. Along with various other lipid parameters, in diabetic females 
total cholesterol was also reported to be higher (Fatima et al., 2016; Khan et al., 2007; Ogbera et al., 2009), the opposite was reported by Uttra et al. (2011). The total cholesterol, alongwith LDL, and triglyceride were also reported to be high in the patients of the periodontitis or non-diabetic patients (Al-Otaibi et al., 2008). The total cholesterol values were increased with increase in age in males, but do not show any correlation with age in females (Shivanand et al., 2012).

High-density lipoprotein cholesterol (mg/dl):

Reference range:30-70 mg/dl

Results are summerized in Fig-3a,b \& 4a,b

Gender and various age dependent comparative highdensity lipoprotein cholesterol ( $\mathrm{mg} / \mathrm{dl})$ for non-diabetic patients: irrespective to the age and gender groups the values of high-density lipoprotein cholesterol were found to fluctuate in between $(37 \pm 0.25$ to $46 \pm 0.32 \mathrm{mg} / \mathrm{dl})$. The further two age groups ( $G-1 \& 2)$ differed statistically from the latter two groups ( $\mathrm{G}-3$ \& 4). Surprisingly, the same picture was not evidenced by the female counterparts. Further, except for the later age-group (G-4), the values obtained for male versus female from the remaining agegroups (group-1, 2 \& 3) differed statistically.

Gender and various age-dependent comparative highdensity lipoprotein cholesterol $(\mathrm{mg} / \mathrm{dl})$ for diabetic patients: irrespective to the age and gender groups the values of High-density lipoprotein cholesterol were found to fluctuate in between ( $21 \pm 0.27$ to $34 \pm 0.25 \mathrm{mg} / \mathrm{dl})$. Except for the age-groups-4 (65-75 yrs) in diabetic female patients, the level of HDL was found to be high as compared to their respective male groups and which were differed statistically from each other. Further, the obtained values of HDL $(\mathrm{mg} / \mathrm{dl})$ in male were found to get decreased with increasing age-groups (G-1 to 4) and were differed statistically from each other. However, in female no such specif ic pattern was evidenced.

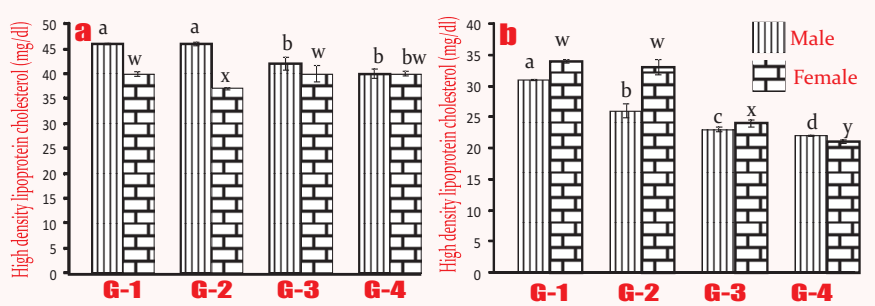

Figure-3: Sex and various age dependant comparative HDLcholestrol for Non-diabetic (a) and diabetic (b) Patients.

Comparative high-density lipoprotein cholesterol (mg/dl) for non-diabetic Versus diabetic tatients (Male): the obtained HDL values of non-diabetic patients were found to be always high and which statistically differed from their respective diabetic counterparts. Further, the HDL values for non-diabetic male patients were found to be higher in earlier two age-groups (G-1 \& 2) and lower in later two agegroups ( $\mathrm{G}-3$ \& 4$)$. Thus the HDL values obtained from the earlier two age-groups did not differ statistically from each other but they differed statistically from the later agegroups. Nevertheless, in diabetic patients, the HDL values revealed a declining trend with respect to the increasing age-groups (G-1 to 4 ) and all the age-groups statistically differed from each other.

Comparative high-density lipoprotein cholesterol (mg/dl) for non-diabetic Versus diabetic Patients (Female): except for the age-groups-2 (46-55 yrs), the remaining obtained HDL values from various age-groups of non-diabetic female females were found to be almost constant and not differ statistically from each other. However, in the female diabetic patients, the HDL values revealed a declining trend only for the two later age-groups, ( $\mathrm{G}-3$ \& 4) and statistically differed from each other and from the earlier two groups. Further, the obtained values for non-diabetic and diabetic patients against each age-group statistically differed from each other.
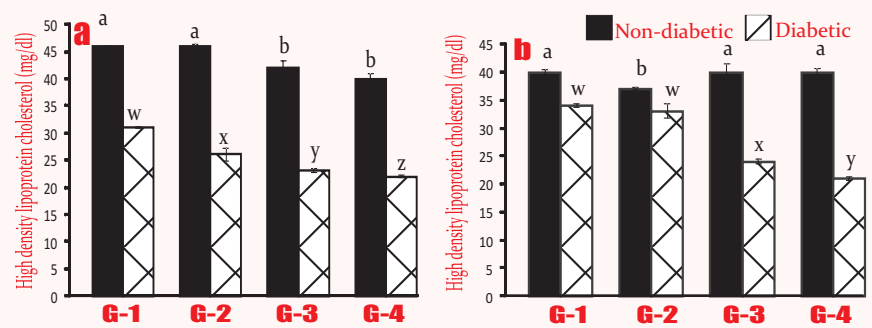

Figure-4: Comparative HDL- Cholestrol for Non-diabetic Versus Diabetic Patients (Male) (a) and (Female) (b)

The HDL level is usually low in diabetic patients as compared to control group (Mahendran et al., 2013). However, a normal HDL in diabetic was also reported by Talat et al. (2003); Sultana (2010). Various studies suggested that HDL cholesterol level has no relation with the age (Ettinger et al., 1992; Garry et al., 1992). However, decrement with age also evident in both males \& females, mainly for the largely middle-aged groups (Berns et al., 1989; Garry et al., 1992) As per the report of DedonderDecoopman, et al.(1980) HDL-cholesterol values were higher in females as compared to the male but got stable with age. Reports also suggest the increase of HDL with age only in men (Ferrara et al., 1997). The lowervalues in female as compared to the male are not a good sign as high HDL level after menopause is cardioprotective. While comparison to Non-diabetic patients, the Lower HDL levels were always evidenced for Diabetic groups for both the genders; males and females (Ali et al., 2015). Further, they also found lower value of this particular parameter in female as compared to males suggesting female are more prone to it (Ali et al., 2015; Khan et al., 2007). The HDL levels were reported to get decreased by $38 \%$ in obese Diabetic patients; whereas by only $26 \%$ in the obese non diabetics (Songa et al., 2013). As per Smith \& Lall (2008) Diabetic male revealed highly significant higher level of cholesterol, LDL cholesterol and triglycerides and 
significantly lower level of HDL cholesterol than the diabetic female whereas Jacobs et al. (2005), Khan et al. (2007), Blebil et al. (2011), Erem et al. (2008) reported significantly higher level of HDL cholesterol among diabetic women.

Triglyceride : Reference range: $\geq 5(\mathrm{mg} / \mathrm{dl})$

Results are summerized in Fig-5a,b \& 6a,b

Gender and various age-dependent comparative triglyceride $(\mathrm{mg} / \mathrm{dl})$ for non-diabetic patients: irrespective to the age and gender groups the values of triglyceride were found to fluctuate in between $(98 \pm 0.92$ to $198 \pm 0.84 \mathrm{mg} / \mathrm{dl}$ ). Further, in the male groups, the triglyceride levels were found to increase constantly with increasing age-groups (G-1 to 4) and were statistically differed from each other. However, upto some extent the same pattern was found in the female groups too except for the latter age-groups (G-4). Interestingly, in the female, except for the age-groups-2 in remaining groups, the triglyceride levels were found to be statistically lower as compared to their respective male groups.

Gender and various age-dependent comparative triglyceride $(\mathrm{mg} / \mathrm{dl})$ for diabetic patients: irrespective to the age and gender groups the values of triglyceride were found to fluctuate in between $(209 \pm 1.42$ to $346 \pm 1.21 \mathrm{mg} / \mathrm{dl}$ ). Further, the levels of triglyceride (mg/dl) were always found to reveal an increasing trend with respect to the increase in age (G-1 to 4 ) and the values were differed statistically from each other. Interestingly, the values of triglyceride in females were always found to be high as compared to their respective male counterpart and which differed statistically from each other.

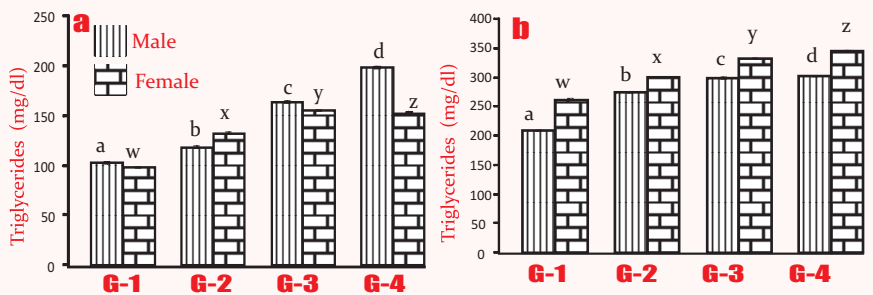

Figure-5: Sex and various age dependant comparative Triglycerides for Non-diabetic (a) and diabetic (b) Patients.

Comparative triglyceride $(\mathrm{mg} / \mathrm{dl})$ for non-diabetic versus diabetic patients (Male): in both the non-diabetic and diabetic male groups the triglyceride $\mathrm{mg} / \mathrm{dl}$ were found to raise continuously with respect to the increasing age ( $\mathrm{G}-1$ to 4) and varied statistically.

Comparative triglyceride (mg/dl) for non-diabetic Versus diabetic Patients (Female): the values for triglyceride for female reflect almost the same as its male counterpart for diabetic patients.

Usually, the mean triglyceride values do not vary between two genders, but a slight increase is evidenced with age (Dedonder-Decoopman, et al., 1980; Albers et al., 1976; Mjos et al., 1977). In our study in both the cases (diabetic and non-diabetic), the trend was on increasing mode with respect to age, perhaps due to their irregular lifestyle and more mental stress (Ali et al., 2015). Further, we found the level of this parameter in a non-diabetic female with respect to the various age-groups almost revealed a low trend of increament than male whereas for diabetic cases the values resembled each other. Almost same report on diabetic females as compared to males was forwarded by Uttra et al. (2011); Firdous \& Khan (2007).

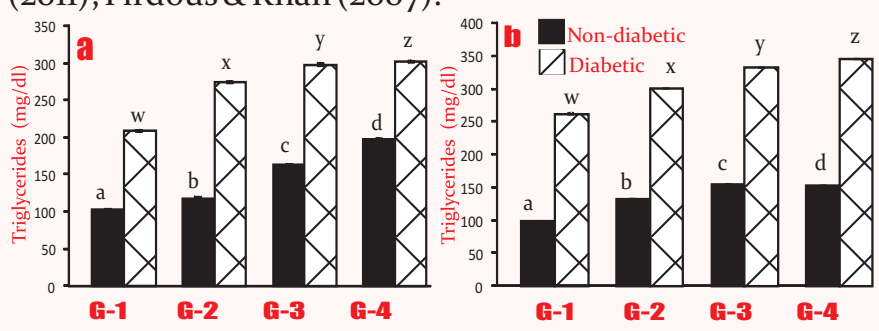

Figure-6: Comparative Triglycerides for Non-diabetic Versus Diabetic Patients (Male) (a) and (Female) (b)

Very Low-density lipoprotein cholesterol (VLDL): Reference range: 10-40 (mg/dl)

Results are summerized in Fig-7a,b \& 8a,b

Gender and various age-dependent comparative VLDL (mg/dl) for non-diabetic patients: irrespective to the age and gender groups the values of VLDL cholesterol were found to fluctuate in between (19.6 \pm 0.18 to $39.6 \pm 0.16$ $\mathrm{mg} / \mathrm{dl})$. Among all the age-groups of male and female the parameters revealed an almost increasing trend with respect to the age-groups and were differed from each other statistically. The obtained values against male and female for each age-groups differ from each other statistically but didn't follow any particular trend for it.

Gender and various age dependent comparative VLDL (mg/dl) for diabetic patients: in all the age and gender groups the obtained values were found to fluctuate in between $(41.8 \pm 0.28$ to $69.2 \pm 0.24 \mathrm{mg} / \mathrm{dl})$. Among all the agegroups of male and female the parameters revealed to increase from earlier age-groups to later age-groups constantly (G-1 to 4) and were statistically differed from each other. In the diabetic patients the VLDL values were found to be more always in various age-groups of female and always differed statistically from their male counterparts.

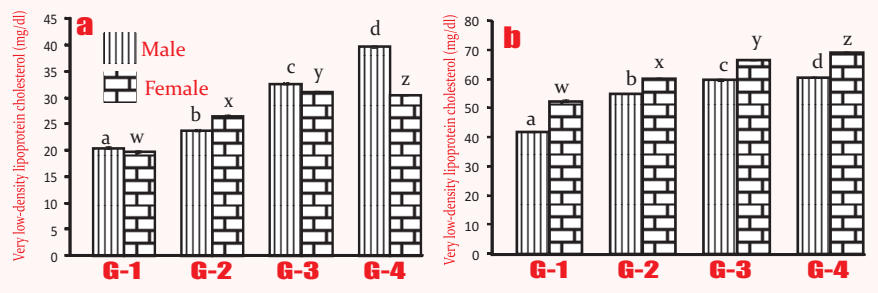

Figure-7: Sex and various age dependant comparative VLDL Cholesterol for Non-diabetic (a) and diabetic (b) Patients.

Comparative VLDL (mg/dl) for non-diabetic versus diabetic patients (Male): the VLDL values were always 
revealed to be high in the diabetic males as compared to the non-diabetic males. However, in both the groups the values of the parameter were found to increase with respect to the increasing age-groups ( $\mathrm{G}-1$ to 4 ) which differed statistically from each other at each age-group.

Comparative VLDL (mg/dl) for non-diabetic versus diabetic patients (Female): the obtained values for female reflect almost the same as its male counterpart for diabetic patients.

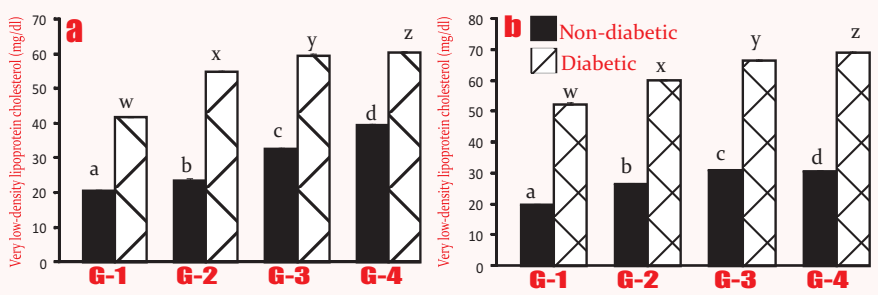

Figure-8: Comparative VLDL Cholesterol for Non-diabetic Versus Diabetic Patients (Male) (a) and (Female) (b)

This parameter indeed corroborates with the other parameters of lipid profile. Our results also strengthened the same, nothing additional remained to discuss in this part.

\section{Low-density lipoprotein cholesterol: \\ Referencerange: $<\mathbf{1 0 0}(\mathrm{mg} / \mathrm{dl})$ \\ Results are summerized in Fig-9a,b \& 10a,b}

Gender and various age-dependent comparative LDL (mg/dl) for non-diabetic patients: irrespective to the age and gender groups the values of LDL-cholesterol were found to fluctuate in between $(82.6 \pm 0.59$ to $160.6 \pm 0.40$ $\mathrm{mg} / \mathrm{dl})$. In both, the groups of the patients, the parameters revealed the lowest value for the earliest age-groups which constantly revealed an increasing trend from the agegroups 1 to 4 .

Gender and various age-dependent comparative LDL (mg/dl) for diabetic patients: irrespective to the age and gender groups the values of LDL were found to fluctuate in between $(127.2 \pm 0.54$ to $206.8 \pm 0.53 \mathrm{mg} / \mathrm{dl})$ and for both the gender groups the parameter revealed an increasing trend with respect to the increasing age-groups (G-1 to 4). Statistically with respect to different gender and various age-groups, each group differed significantly from its counterpart and consecutive groups.

Comparative LDL (mg/dl) for non-diabetic versus diabetic patients (Male): with respect to the various age-groups, the LDL values were always revealed to be high in the diabetic patients as compared to the non-diabetic patients. However, in both the groups the values of the parameter were found to increase with respect to the increasing agegroups (G-1 to 4) which differed statistically from each other.

Comparative LDL (mg/dl) for non-diabetic versus diabetic Patients (Female): the obtained values for the females reflect almost the same as its male counterpart for diabetic patients.

The concentration of low-density lipoprotein cholesterol $(\mathrm{mg} / \mathrm{dl})$ was already reported to be influenced as per age and gender (Abbott et al., 1983; Grundy et al., 1985; Millar et al., 1995). Women at early stage of life (premenopausal stage) evidenced lower LDL and VLDL but higher levels of anti- HDL (Dedonder-Decoopman et al., 1980). Almost the same picture was reported by Ferrara et al. (1997) in their study but only in male not in female. The levels of TC and LDL-C were signif icantly higher in female as compared to male in diabetic patients were reported by various workers (Assmann et al., 1983; Friedewald et al., 1972; Wexler et al., 2005). Various reports suggested that after an age of 65 years total and LDL cholesterol levels decrease with age (Ettinger et al., 1992; Frishman et al., 1992; Garry et al., 1992). In each age-group, values were found to be more in diabetic male as compared to females by Uttra et al. (2011).

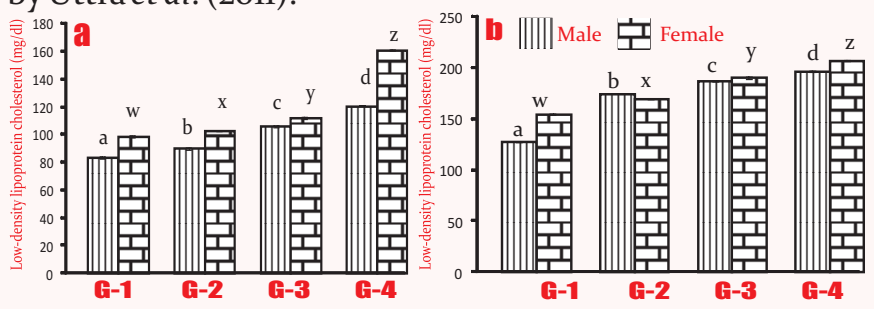

Figure-9: Sex and various age dependant comparative LDL Cholesterol for Non-diabetic (a) and diabetic (b) Patients.
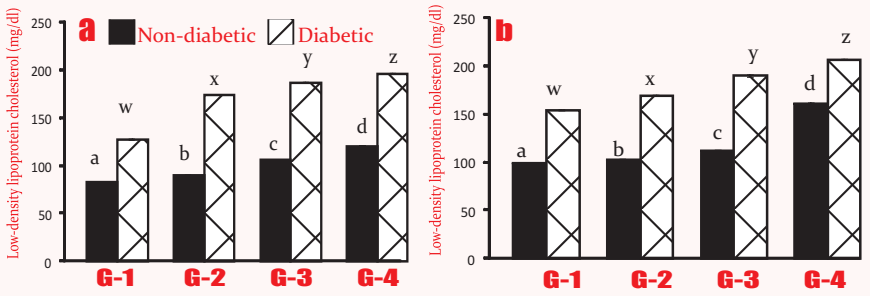

Figure-10: Comparative LDL Cholesterol for Non-diabetic Versus Diabetic Patients (Male) (a) and (Female) (b)

\section{Conclusion:}

Our study indicates that in the population of Raipur province there is comparatively more dominance of disorders in lipid profile among the patients of diabetes. A Positive relationship with increament in the lipid profile values between age and gender have been strongly evidenced. The disorders related to lipids occurred more frequently in the aged diabetic females of Raipur. The existing condition in the female may be due to imbalances and disturbances in the hormonal secretions. Perhaps, Raipur is a growing metropolitan city and due to which irregularities in the lifestyle suddenly increased, further, abrupt changes in the feeding habits and less availability of time due to the busy schedule in daily life like other metro cities resultant to such conditions in the inhabitants. The situation created suddenly and perhaps the female 
populations are yet needed time to cope properly with the situation. However, the occurrences of abnormal or high lipid values including low levels of HDL Cholesterol which is also good cholesterol might be playing a crucial role in the advancement of disorders related to the cardiovascular system. Thus the diabetic females of Raipur are more prone towards the progression of cardiovascular diseases in the future.

\section{Acknowledgment:}

Authors thankful to all the members of the Clinical Path Lab $(\mathrm{CPL})$ group who have immensely contributed in my research and was a source of guidance, friendship and collaboration. In this series I personally thank Dr. P.K. Khodiar, Associate Professor, Department of Biochemistry, Pt. Jawahar Lal Nehru Memorial Medical College, Raipur, Mr. Sanjay Dharmraj, Director, CPL, and Ms. Sandhyarani P, Lab supervisor, CPL for their enthusiasm and willingness that helped me to carryout various tests related to my study..

\section{References:}

Abbott, R.D., Garrison, R.J., Wilson, P.W., Epstein, F.H., Castelli, W.P., Feinleib, M. \& LaRue, C. (1983): Joint distribution of lipoprotein cholesterol classes. The Framingham study. Arterioscl. Throm. Vasc. Bio., 3(3):260-272.

ADA (2009): Diagnosis and Classification of Diabetes Mellitus. Diabetes Care, $32(\mathrm{~S}-1): \mathrm{S} 62 \mathrm{~S} 67$.

Albers, J.J., Wahl, P.W., Cabana, V.G., Hazzard, W.R. \& Hoover, J.J., (1976): Quantitationof apolipoprotein, A-I of human Plasma high density lipoprotein. Metabolism, 25(6):633-644.

Ali, F., Jamil, H., Anwar, S.S. \& Wajid, N. (2015): Characterization of lipid parameters in diabetic and non-diabetic atherosclerotic patients. L. Geriatr. Cardiol., 12(1):37-43.

Al-Otaibi, D.H., Babay, N.A., Habib, S.S. \& Almas, K. (2008): Assessment of lipid profile in Saudi type 2 diabetic and nondiabetic periodontal patients. Saudi Med. J., 29(5):723-727.

Assmann, G., Schriewer, H., Schmitz, G. \& Hägele, E.O. (1983): Quantification of high-density-lipoprotein cholesterol by precipitation with phosphotungstic acid $/ \mathrm{MgCl}_{2}$. Clin. Chem., 29:2026-2030.

Austin, M.A. \& Edwards, K.L. (1996): Small, dense low density lipoproteins, the insulin resistance syndrome and noninsulindependent diabetes. Curr. Opin. Lipidol., 7(3):167-171.

Bartels, D.W., Davidson, M.H. \& Gong, W.C. (2007): Type 2 diabetes and cardiovascular disease: reducing the risk, $L$ Mana. Care Phar., 13 (2 S-A):S2-S15.

Berns, M.A.M., de Vries, J.H.M. \& Katan, M.B. (1989): Increase in body fatness as a major determinant of changes in serum total cholesterol and high density lipoprotein cholesterol in young men overa 10-year period. Am. J. Epidemiol., 130(6):1109-1122.

Blebil, A.Q., Hassan, Y. \& Dujaili, J.A. (2011): Differences in demographics, lipid prof ile and other clinical characteristics among type 2 diabetic patients in the state of penang, malaysia according to gender and races. Asian J. Pharmac. Clin. Res., 4(2):130-133.

Dansethakul, P., Thapanathamchai, L., Saichanma, S., Worachartcheewan, A., and Pidetcha, P. (2015): Determining a new formula for calculating low-density lipoprotein cholesterol: data mining approach. EXCLI Journal, 14:478483.

Dedonder-Decoopman, E., Fievet-Desreumaux, C., Campos, E., Moulin, S., Dewailly, P., Sezille, G. and Jaillard, J. (1980): Plasma levels of VLDL- + LDL-cholesterol, HDL-cholesterol, triglycerides and apoproteins B and A-I in a healthy population--influence of several risk factors. Atherosclerosis, 37(4):559-568.

Erem, C., Hacihasanoglu, A., Deger, O., Kocak, M. and Topbas, M. (2008): Prevalence of dyslipidemia and associated risk factors among Turkish adults: Trabzon lipid study. Endocrine, 34(13):36-51.

Ettinger, W.H.., Wahl, P.W., Kuller, L.H., Bush, T.L., Tracy, R.P., Manolio, T.A., Borhani, N.O., Wong, N.D. and O'Leary, D.H. (1992): Lipoprotein lipids in older people. Results from the Cardiovascular Health Study. The CHS Collaborative Research Group. Circulation, 86(3):858-869.

Farmer, J.A. (2007): Diabetic dyslipidemia and atherosclerosis: Evidence from clinical trials. Current Atherosclerosis Reports, 9(2):162-168.

Fatima, S., Ijaz, A., Sharif, T.B., Khan, D.A. and Siddique, A. (2016): Accuracy of non-fasting lipid profile for the assessment of lipoprotein coronary risk. Journal of the College of Physicians and Surgeons-Pakistan. 26(12):954-957.

Ferrara, A., Barrett-Connor, E. and Shan, J. (1997): Total, LDL, and HDL cholesterol decrease with age in older men and women. The Rancho Bernardo Study 1984-1994. Circulation, 96(1):3743.

Firdous, S. and Khan, M.Z. (2007): Comparison of patterns of lipid prof ile in type-2 diabetics and non-diabetics. Annals of King Edward Medical University, 3(1):84-87.

Friedewald, W.T., Levy, R.I. and Fredrickson, D.S. (1972): Estimation of the concentration of low-density lipoprotein cholesterol in plasma without use of the preparative ultracentrifuge. Clinical Chemistry, 18(6):499-502.

Frishman, W.H., Ooi, W.L., Derman, M.P., Eder, H.A., Gidez, L.I., Ben-Zeev, D., Zimetbaum, P., Heiman, M. \& Aronson, M. (1992): Serum lipids and lipoproteins in advanced age. Intraindividual changes. Ann. Epidemiol., 2(1-2):43-50.

Garry, P.J., Hunt, W.C., Koehler, K.M., Vander Jagt, D.J. \& Vellas, B.J. (1992): Longitudinal study of dietary intakes and plasma lipids in healthy elderly men and women. Am. J. Clin. Nutr. 55(3):682-688.

Ghadge, A.A., Diwan, A.G., Harsulkar, A.M. \& Kuvalekar, A.A. (2017): Gender dependent effects of fasting blood glucose levels and disease duration on biochemical markers in type 2 diabetics: A pilot study. Diabetes Metab. Synd.: Clinical Res. Rev., $11(\mathrm{~S}-1): \mathrm{S}_{4} 81-\mathrm{S}_{4} 89$.

Ghazanfari, Z., Niknami, S., Ghofranipour, F., Larijani, B., AghaAlinejad, H. and Montazeri, A. (2010): Determinants of glycemic control in female diabetic patients: a study from Iran. Lipids Health Dis., 9:83.

Grundy, S.M., Vega, G.L., \& Bilheimer, D.W. (1985): Kinetic Mechanisms Determining Variability in Low Density Lipoprotein Levels and Rise with Age. Arteriosclerosis, 5(6):623-63o.

Grundy, S.M., Cleeman, J.I., Daniels, S.R., Donato, K.A., Eckel, 
R.H., Franklin, B.A., Gordon, D.J., Krauss, R.M., Savage, P.J., Smith, Jr, S.C., Spertus, J.A. \& Costa, F. (2005): Diagnosis and management of the metabolic syndrome: An American Heart Association/National Heart, Lung, and Blood Institute Scientif ic Statement. Circulation, 112(17):2735-2752.

Gupta, R., Sarna, M., Thanvi, J., Sharma, V. and Gupta, V.P. (2007): Fasting glucose and cardiovascular risk factors in an urban population. L. Asso. Physici. India, 55:705-709.

Hussain, A., Vaaler, S., Sayeed, M.A., Mahtab, H., Ali, S.M. \& Khan, A.K. (2007): Type 2 diabetes and impaired fasting blood glucose in rural Bangladesh: a population-based study. Eur. J. Pub. Health, 17(3):291-296.

Jacobs, M., Kleisli, T., Pio J, Malik, S., L'Italien, G.J., Chen, R.S. \& Wong, N.D. (2005): Prevalence and control of dyslipidemia among persons with diabetes in the United States. Diabetes Res. Clin. Pr. 70(3):263-269.

Kaveeshwar, S.A. \& Cornwall, J. (2014): The current state of diabetes mellitus in India. Aust. Med. J., 7(1):45-48.

Khan, H.A., Sobki, S.H. \& Khan, S.A. (2007): Association between glycaemic control and serum lipids prof ile in type 2 diabetic patients: HbAic predicts dyslipidaemia. Clin. Exp. Med., 7(1):24-29.

Khaw, K.T., Wareham, N., Bingham, S., Luben, R., Welch, A. \& Day. N. (2004): Association of hemoglobin Aic with cardiovascular disease and mortality in adults: The European prospective investigation into cancer in Norfolk. Ann. Int. Med., 141(6):413-420.

Ko, G.T.C., Wai, H.P.S. \& Tang, J.S.F. (2006): Effects of Age on Plasma Glucose Levels in Non-diabetic Hong Kong Chinese. Croat. Med.J., 47(5):709-713.

Krauss, R.M. (2004): Lipids and lipoproteins in patients with type 2 diabetes. Diabetes Care, 27(6):1496-1504.

Mahendran, B.K., Santha, K., Santhoshkumar, N., Desigan, G.E. \& Anwar, M. (2013): Serum Homocysteine And Lipid Profile Levels In Type 2 Diabetes Mellitus Patients. Int. J. Med. Res. Health Sci., 2(2):200-205

Manoria, P. C., Chopra, H. K., Parashar, S. K., Dutta, A. L., Pinto, B., Mullasari, A. \& Prajapati, S. (2013): The nuances of atherogenic dyslipidemia in diabetes: Focus on triglycerides and current management strategies. Indian Heart J., 65(6):683-69o.

Millar, J.S., Lichtenstein, A.H., Cuchel, M., Dolnikowski, G.G., Hachey, D.L., Cohn, J.S. \& Schaefer, E.J. (1995): Impact of age on the metabolism of VLDL, IDL, and LDL apolipoprotein B100 in men. J. Lipid Res., 36(6):1155-1167.

Mjos, O.D., Thelle, D.S., Forde, O.H. and Vik-Mo, H. (1977): Family Study of High Density Lipoprotein Cholesterol and the Relation to Age and Sex. J. Int. Med., 201(1-6):323-329.

Motala, A.A., Omar, M.A. \& Pirie, F.J. (2003): Diabetes in Africa. Epidemiology of type 1 and type 2 diabetes in Africa. $L$. Cardiovas. Risk, 10(2):77-83.

Nigam, P.K., (2014): Calculated Low Density Lipoprotein-Cholesterol: Friedewald's Formula versus Other Modif ied Formulas: Calculated LDL Cholesterol. Int. J.Life Sci.Med. Res., 4(2):25-31.

Ogbera, A.O., Fasanmade, O.A., Chinenye, S., \& Akinlade, A. (2009): Characterization of lipid parameters in diabetes mellitus-a Nigerian report. Int. Arch. Med., 2:19.
Ozder, A. (2014): Lipid profile abnormalities seen in T2DM patients in primary healthcare in Turkey: a cross-sectional study. Lipids Health Dis., 13:183.

Schofield, J.D., Liu, Y., Rao-Balakrishna, P., Malik, R.A. \& Soran, H. (2016): Diabetes Dyslipidemia. Diabetes Ther., 7(2):203-219.

Seuring, T., Archangelidi, O. and Suhrcke, M. (2015): The Economic Costs of Type 2 Diabetes: A Global Systematic Review. PharmacoEconomics, 33(8):811-831.

Shivanand, K.G., Manjunath, M.L. \& Jeganathan, P.S. (2012): Lipid profile and its complications in diabetes mellitus. Int. J. Biomed.Adv. Res., 03(10):775-78o.

Smith, S. \& Lall, A.M. (2008): A Study on Lipid Profile Levels of Diabetics and Non-Diabetics Among Naini Region of Allahabad, India. Türk Biyokimya Dergisi, 33(4):138-141.

Songa, R.M., Siddhartha, K. \& Sudhakar, K. (2013): Lipid prof ile in type 2 diabetes mellitus with obesity. Bull. Pharma. Med. Sci., 1(2):132-138.

Sudha. K., Ashok, P. K., Hegde, A., Marathe, A. and Kiran, K.A.M. (2015): Effect of serum triglycerides on LDL estimation by Friedewald formula and direct assay: A laboratory based study. Int. J. Biomed. Res., 6(03):189-191.

Sultana, R. (2010): Impact Of Duration OfType 2 Diabetes Mellitus On Lipid Prof ile. Gomal J. Med. Sci., 8(1):57-59.

Talat, N., Khan, A., Gulsena, M., Bilal, B-Y. \& Chaudhry, M.A. (2003): Dyslipidemias in Type II Diabetes Mellitus Patients in a Teaching Hospital of Lahore, Pakistan. Paki. J. Med. Sci., $19(4): 283-286$.

Tenenbaum, A., Fisman, E.Z., Motro, M. \& Adler, Y. (2006): Atherogenic dyslipidemia in metabolic syndrome and type 2 diabetes: therapeutic options beyond statins. Cardiovas. Diabetol., 5:20.

Trevisan, R., Dodesini, A.R. \& Lepore, G. (2006): Lipids and Renal Disease.J.Am. Soc. Nephrol., 17(4 S- 2):S145-S147.

Turner, R., Cull, C. \& Holman, R. (1996): United Kingdom Prospective Diabetes Study 17: a 9-year update of a randomized, controlled trial on the effect of improved metabolic control on complications in non-insulin-dependent diabetes mellitus. Ann. Intern. Med., 124(1 Pt 2):136-145.

Uttra, K.M., Devrajani, B.R., Shah, S.Z.A., Devrajani, T., Das, T., Raza, S. \& Naseem (2011): Lipid Prof ile of Patients with Diabetes mellitus (A Multidisciplinary Study). World App. Sci. J., 12 (9):1382-1384.

Valensi, P., Avignon, A., Sultan, A., Chanu, B., Nguyen, M.T. \& Cosson, E. (2016): Atherogenic dyslipidemia and risk of silent coronary artery disease in asymptomatic patients with type 2 diabetes: a cross-sectional study. Cardiovas. Diabetol., 15(1):104.

Veghari, G., Sedaghat, M., Joshaghani, H., Banihashem, S., Moharloei, P., Angizeh, A., Tazik, E., Moghaddami, A., Hajian-Tilaki, K. \& ZahedPasha, Y., (2014): The association of fasting blood glucose (FBG) and waist circumference in northern adults in Iran: a population based study. J. Diabetes Metab. Disord., 13(1):2.

Wexler, D.J., Grant, R.W., Meigs, J.B., Nathan, D.M. and Cagliero, E. (2005): Sex disparities in treatment of cardiac risk factors in patients with type 2 diabetes. Diabetes Care, 28(3):514-520. 